\title{
Electrowetting on curved surfaces
}

\author{
Ying Wang and Ya-Pu Zhao* \\ Received 1st October 2011, Accepted 5th December 2011 \\ DOI: $10.1039 / \mathrm{c} 2 \mathrm{sm} 06878 \mathrm{~h}$
}

In this work, we explore the influence of the surface curvature on the contact angle in electrowetting (EW). By using the method of energy minimization, an extended EW equation for EW on curved surfaces is obtained. A curvature-modified EW number is introduced to describe the surface curvature effect. It is found that the variation of the contact angle will be curvature dependent; especially, with decreasing of the system size, the surface curvature effect would be even more significant. Comparing with the planar situation, the contact angle variation decreases on convex surfaces but increases on concave surfaces. Then considering the line tension effect, an extended expression for EW on curved surfaces is proposed. Since curved surfaces are more common, both natural and synthetic, our findings will have important implications for the miniaturization of future electronic products.

\section{Introduction}

Microfluidic systems are of great importance in various fields, such as 'lab-on-a-chip' devices, ${ }^{1,2}$ cell assays in microfluidics, ${ }^{3}$ and adjustable lenses. ${ }^{4}$ Electrowetting (EW) or electrowetting on dielectric (EWOD), as an important method of controlling the liquid droplet, has attracted considerable attentions, and has been developing fast in recent decades. EW is used to change the wettability of the liquid droplet on a solid substrate by applying an electric field. Earlier studies and applications mainly concentrated on planar surfaces, and have scored great success. However, since EWOD on planar surfaces is a specific and relatively simple situation, structured surfaces with curved features are more common, such as liquid lenses with curved contact surfaces, ${ }^{5}$ flexible paper-like substrates, ${ }^{6,7}$ and Lotus leaves. ${ }^{8}$ Besides, EWOD on curved surfaces is a promising candidate for a variety of potential applications such as electronic display technique, ${ }^{9}$ low-cost and more flexible e-paper substrate, ${ }^{10}$ and nanoflow in carbon nanotubes (CNTs). ${ }^{11,12}$ As a good candidate for the drug delivery on micro- and nanoscales, the study of electro-elasto-capillary (EEC) is of great significance. Yuan and $\mathrm{Zhao}^{13}$ simulated an EEC process in which wrapping and unwrapping of a droplet could be dynamically controlled. During the dynamic EEC process, the curvature of the graphene substrate was variable.

In 1875, Lippmann ${ }^{14}$ discovered the new phenomenon that the capillary climb of mercury changed by introducing a voltage between the mercury and the electrolyte solution, which is called electrocapillarity. The explanation of this phenomenon was that the induced residual charge changed the solid-liquid surface tension $\gamma_{S L}$, and the Lippmann equations were put forward:

State Key Laboratory of Nonlinear Mechanics (LNM), Institute of Mechanics, Chinese Academy of Sciences, Beijing, 100190, China. E-mail:yzhao@imech.ac.cn.

$$
\begin{gathered}
\sigma_{e}=-\frac{\partial \gamma_{S L}}{\partial V}, \\
C_{H}=\frac{\partial \sigma_{e}}{\partial V},
\end{gathered}
$$

where $\sigma_{e}$ is the surface charge density, $V$ is the value of the applied voltage, $C_{H}$ is the capacitance per unit area. The electrocapillarity phenomenon and the Lippmann equations are the basis of current EW. In the following centuries, people kept studying this phenomenon, ${ }^{15,16}$ from electrocapillarity to EW. As in early studies of EW, the liquid droplet was directly deposited on the metal substrate, and electrolytic decomposition took place when the applied voltage went up to a few hundred millivolts. ${ }^{17}$ So, it was hard to put into an application. This problem was solved by placing a thin dielectric film on the substrate to separate it from the liquid droplet. ${ }^{18,19}$ The applied voltage can hence be increased to hundreds of volts. Berge ${ }^{18,19}$ carried out such experiments in the 1990s, and gave the electrowetting (EW) equation by combining the Lippmann equations with the Young equation:

$$
\cos \theta(V)=\cos \theta_{0}+\frac{\varepsilon V^{2}}{2 \gamma_{L V} d},
$$

where $\theta(V)$ is the contact angle under a certain voltage, $\theta_{0}$ is the contact angle without applied voltage, $\varepsilon$ is the dielectric constant, $d$ is the thickness of the dielectric film. The last term of eqn (3) is a dimensionless number, which is called the EW number:

$$
\eta=\frac{\varepsilon V^{2}}{2 \gamma_{L V} d} .
$$

The EW number represents the ratio of the electrostatic energy to the liquid-vapor interfacial energy. ${ }^{20}$ As the capacitance of a parallel-plate capacitor per unit area is $c=\varepsilon / d$, eqn (3) can also be expressed as 


$$
\cos \theta(V)=\cos \theta_{0}+\frac{c V^{2}}{2 \gamma_{L V}} .
$$

The EW equation is the fundamental of EWOD.

The mechanism of the contact angle reduction as a result of the applied voltage is important for the understanding of the EWOD phenomenon. However, the mechanism is still debatable nowadays. Possible interpretations are summarized in Fig. 1. According to Lippmann, ${ }^{14}$ the solid-liquid interfacial energy changed when the voltage was applied. This is known as the electrocapillarity. This assertion was disputed by Gupta and coworkers, ${ }^{21}$ for they conducted experiments and found that the solid-liquid interfacial energy was invariable. In contrast, an explanation of secondary electrocapillarity was proposed by Digilov, ${ }^{22}$ who suggested that the applied potential caused polarization of the three-phase contact line, leading to a change in the line tension and as thereby in the contact angle. Effective capacitance, in which the dielectric film is taken as part of the solid-liquid interface, ${ }^{17}$ is also one of the interpretations. The dielectric film works as a capacitor and almost all of the electric energy is stored in it. From the viewpoint of physical mechanics, the reduction of the contact angle may result from the repulsion

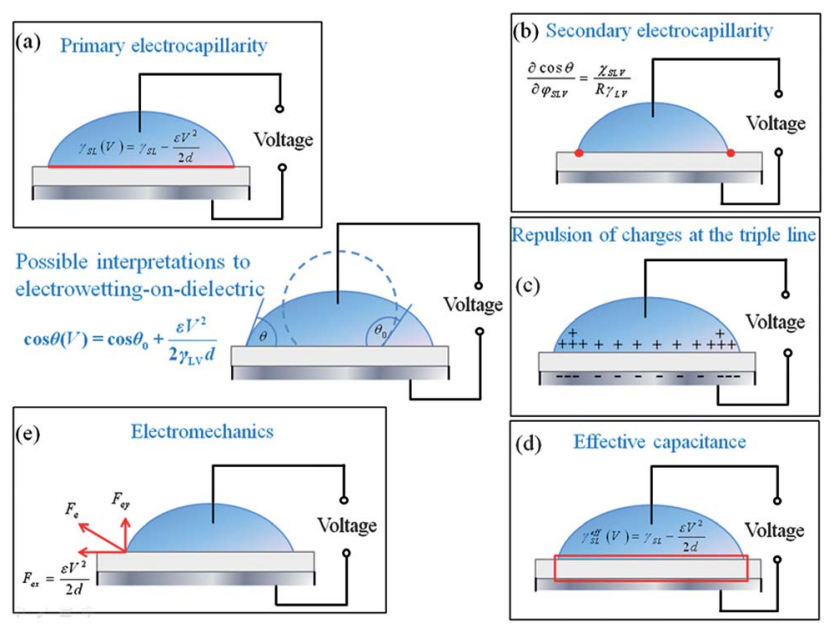

Fig. 1 Possible interpretations (a)-(e) to the reduction of the contact angle (CA) as a result of the applied voltage: primary electrocapillarity, secondary electrocapillarity, repulsion of like-charges at the triple line, effective capacitance and electromechanics. (a) Primary electrocapillarity means that the solid-liquid interfacial energy changes by applying a voltage, i.e., $\gamma_{S L}(V)=\gamma_{S L}-\frac{\varepsilon V^{2}}{2 d}$, thus leading the reduction of CA. (b) Secondary electrocapillarity indicates that the applied voltage causes polarization of the triple line, leading to change in the line tension and as thereby in CA, i.e., $\frac{\partial \cos \theta}{\partial \varphi_{S L V}}=\frac{\chi_{S L V}}{R \gamma_{L V}}$, where $\chi_{S L V}$ is the line density of the electric charges and $\varphi_{S L V}$ is the electrostatic potential on the triple line. (c) Repulsion of the like-charges at the triple line shows that it is the repulsion that may cause the contact line moves and droplet spreads. (d) Effective capacitance shows that the dielectric film is taken as part of the solid-liquid interface. The effective solid-liquid interfacial energy changes as the voltage is applied, i.e., $\gamma_{S L}^{e f f}(V)=\gamma_{S L}-\frac{\varepsilon V^{2}}{2 d}$. The dielectric film works as a capacitor and almost all of the electric energy is stored in it. (e) Electromechanics illustrates that the reduction of CA is a result of the Maxwell stress tensor or the electrostatic force. of like-charges at the triple line. Electromechanics is an interpretation that widely accepted today. Jones ${ }^{23}$ addressed EWOD as an electromechanical phenomenon within the framework of electromechanics. This point of view was supported by Kang, ${ }^{24}$ who suggested that the EWOD phenomenon originated from the electrostatic pressure by using the classical electrostatics, namely it is the Maxwell tensor that "drags" the droplet to spread out. Mugele also believed the contact angle reduction is the result of the electrostatic forces pulling on the drop surface. ${ }^{25} \mathrm{Up}$ to now it is still dubious which mechanism dominates in EWOD, though these interpretations seem to be related in a sense. Whether one of the above interpretations is the reason or several mechanisms work together is still unclear, and more investigations are necessary. Since the mechanism has been studied by researchers from various fields, the EW equation can be derived from different approaches, such as the thermodynamic approach, the energy minimization approach, and the electromechanical approach.

The EW equation was derived from EWOD on planar surfaces, and was in good accordance with the experimental results. Then the question comes, is it applicable for EWOD on curved surfaces? Though experiments of EWOD on curved surfaces have been studied for years and put into application, the theory is still lacking. Kim and Steckl ${ }^{10}$ studied the feasibility of using paper as a cheap and flexible substrate for e-paper, electronic display and other EW devices. In their study, they compared EWOD on rolled-paper substrate with different materials and used the EW equation as the theory reference. Fan and co-workers ${ }^{26}$ studied "droplet-on-a-wristband" based on the study of EWOD in curved devices. They conducted experiments on curved surfaces whose curvatures were $0,0.02,0.04$ and 0.06 $\mathrm{mm}^{-1}$, respectively, and found that the curvature had no noticeable influence on the required driving voltages at the center point. Wheeler's group ${ }^{27}$ explored all-terrain droplet actuation (ATDA). They built the ATDA model and conducted relevant experiments. However, in their theoretical expression of the EW force, the surface curvature effect was not considered. While the EW equation was directly used to interpret and predict EWOD on curved surfaces in the above studies, some other groups considered the influence of the curvature. Chen ${ }^{28}$ studied EW in CNTs by the means of molecular dynamics (MD) simulations. They considered the influence of the surface curvature and adopted Lippmann's model of EW for a cylindrical capacitor. According to their research, the MD simulations were in good agreement with the theory.

In the EW equation, when the voltage $V$ equals to zero, i.e., without applied voltage, the initial contact angle $\theta_{0}$ on planar surfaces can be obtained by using the Young equation..$^{29}$ Extrand and Moon ${ }^{30}$ conducted a series of experiments of wetting on spherical surfaces. In their experiments, water was deposited on the flat and spherical surfaces with polytetrafluoroethylene (PTFE) and polycarbonate (PC) coatings, respectively. The diameters of the spheres ranged from $3.2 \mathrm{~mm}$ to $25.4 \mathrm{~mm}$. They found that the contact angles on the spheres are the same as that on planar surfaces. The Young equation was proved to comply with the experimental results on spherical surfaces. Here on spherical surfaces, the contact angle $\theta$ is defined as the angle between the solid-liquid surface tangent and the liquid-vapor surface tangent, as illustrated in Fig. 2. However, on and below 
(a)

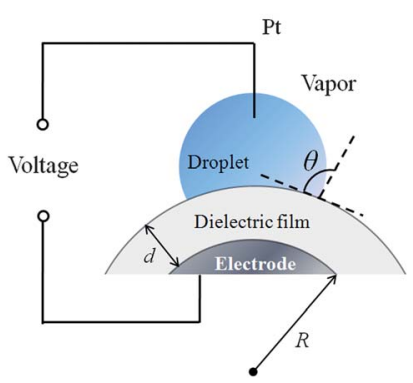

(b)

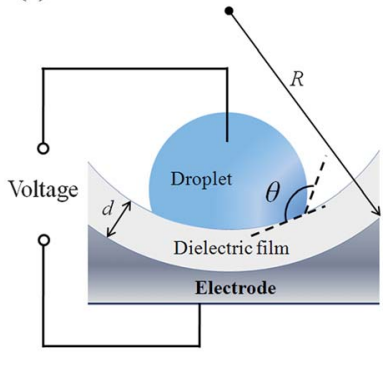

Fig. 2 Schematic of EWOD on curved surfaces: (a) EWOD on a convex surface; (b) EWOD on a concave surface. A conducting liquid droplet is placed on a metal counter electrode coated with a dielectric film. The contact angle $\theta$ changes when a voltage is induced.

the microscale, the line tension will play an important role to determine the wetting behavior of the droplet. The contact angle on a curved surface is different from that on a planar one ${ }^{31,32}$ and the Young equation should be modified. ${ }^{33}$ Considering the surface roughness, Bormashenko ${ }^{34}$ proposed a modified Young equation for wetting on rough curved surfaces. The line tension effect was also discussed.

While the initial contact angle on curved surfaces without voltage has been studied, the variation of the contact angle on a curved substrate under certain voltage is still ambiguous. Therefore, in this work, the influence of the surface curvature on the contact angle in EWOD is investigated, by using the principle of energy minimization. Since a curved surface could be either convex or concave, the curvature is considered as an algebraic value (i.e., $R<0$ for the concave surface, while $R>0$ for the convex surface), and a uniform expression is obtained. Afterwards, we discuss how surface curvature influences the EWOD process and analyze how large the surface curvature influences the variation of the contact angle. It is found that the surface curvature effect would be significant and the EW equation should be modified on and below the microscale.

\section{Theoretical model for consideration of surface curvature}

Compose a thermodynamic system by the droplet, the dielectric film, the curved metal counter electrode, and the voltage source, as shown in Fig. 2. The contact angle $\theta$ is the angle between the two tangents at the three-phase contact line, just as the usual definition on a curved surface. ${ }^{30}$ The following derivation is based on an ideal situation, such as the liquid droplet is a perfect conductor and deposited on the substrate symmetrically, the contact angle hysteresis is neglected. When the droplet spreads, the free energy $F$ of the system changes. The increment of $F$ can be taken as:

$$
d F=\gamma_{S L} d A-\gamma_{S V} d A+\gamma_{L V} d A \cos \theta+d U-d W_{B},
$$

where $\gamma_{S L}, \gamma_{S V}$ and $\gamma_{L V}$ are the solid-liquid, solid-vapor, and liquid-vapor interfacial energy, respectively. $U$ is the electric energy, $W_{B}$ is the work that the voltage source performs, $A$ is the droplet base area (Fig. 3).

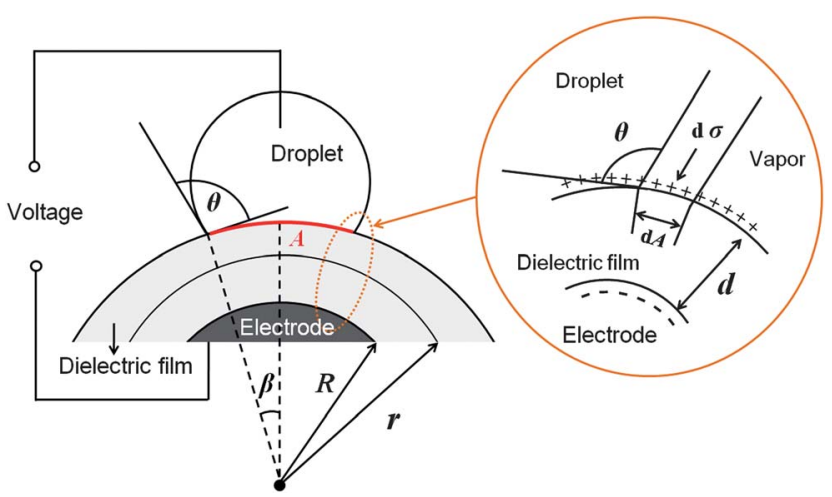

Fig. 3 Model for calculating the electric field intensity $E$. The inset depicts the variation of the contact angle as a result of the applied voltage. When the voltage is applied, the droplet base area increases to reach a new equilibrium.

A droplet can stay stable on a surface when its free energy reaches a minimum, namely $d F=0$. Divide $\mathrm{d} F$ by $\mathrm{d} A$, and make it equal to 0 , eqn (6) is then

$$
\gamma_{S L}-\gamma_{S V}+\gamma_{L V} \cos \theta+\frac{d U}{d A}-\frac{d W_{B}}{d A}=0 .
$$

In order to get the contact angle $\theta$, we should know the values of $\mathrm{d} U / \mathrm{d} A$ and $\mathrm{d} W_{\mathrm{B}} / \mathrm{d} A$.

According to the electromagnetics, $U$ can be taken by the formula $d U=\frac{1}{2} \varepsilon E^{2} d v$, where $E$ is the electric field intensity, and $v$ is the volume filled with an electric field. The predominant effect in the EWOD process is the distribution of the electric field, which is dependent on the structure of the metal counter electrode coated with a dielectric film. The distribution of the electric field is very complicated near the three-phase contact line. For simplicity but without loss of generality, in the following derivation we suppose the area with the electric field singularity be much smaller compared with that in the uniform field, and use expressions with an average value effect.

\section{EWOD on spherical surfaces}

In the case of EWOD on a convex spherical surface (radius is $R$ ) coated with a dielectric film with thickness of $d$, when the droplet spreads with an infinitesimal base area increment $\mathrm{d} A$, the volume increment $\mathrm{d} v$ under the droplet base can be calculated:

$$
d v=\int_{R}^{R+d} \frac{d A}{(R+d)^{2}} r^{2} d r,
$$

where $r$ is the radius of the sphere between the dielectric-vapor surface and the dielectric-metal surface, as depicted in Fig. 3. Then we can get the expression of $\mathrm{d} U / \mathrm{d} A$ :

$$
\frac{d U}{d A}=\int_{R}^{R+d} \frac{1}{2}\left\langle\varepsilon E^{2}\right\rangle \frac{r^{2}}{(R+d)^{2}} d r .
$$

Here, the angle brackets denote the average over $r$, i.e., the average value of the electric field intensity $E$ is only the function of $r$. 
In a partial spherical capacitor, we can get the area under the droplet base with the radius of $r$ :

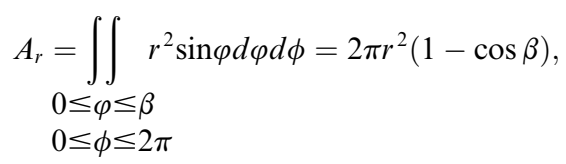

where $\beta$ is half the opening angle of the droplet on the base. Using the Gauss' flux theorem:

$$
\begin{gathered}
\langle E(r)\rangle=\frac{Q}{\varepsilon A_{r}}=\frac{Q}{2 \pi \varepsilon r^{2}(1-\cos \beta)}, \\
V=\int_{R}^{R+d}\langle E(r)\rangle d r=\frac{Q}{2 \pi \varepsilon(1-\cos \beta)}\left(\frac{1}{R}-\frac{1}{R+d}\right),
\end{gathered}
$$

where $Q$ is the quantity of electric charge. Substituting eqn (11) into eqn (12), we can get the relationship between the applied voltage and the electric field intensity

$$
\langle E(r)\rangle=\frac{R(R+d)}{d r^{2}} V .
$$

Then eqn (9) can be rewritten as

$$
\frac{d U}{d A}=\frac{\varepsilon V^{2}}{2 d} \cdot \frac{R}{R+d}
$$

In the droplet spreading process, the work per unit area $\mathrm{d} W_{\mathrm{B}} /$ $\mathrm{d} A$ done by the voltage source to redistribute the charge can be written as

$$
\frac{d W_{B}}{d A}=V \sigma=V \varepsilon E_{R+d}=\frac{\varepsilon V^{2} R}{(R+d) d},
$$

in which $\sigma$ is the surface charge density. With eqns (14) and (15), calculating the minimum of the free energy:

$$
\gamma_{S L}-\gamma_{S V}+\gamma_{L V} \cos \theta+\frac{\varepsilon V^{2}}{2 d} \cdot \frac{R}{R+d}-\varepsilon V^{2} \frac{R}{d(R+d)}=0 .
$$

The Young equation $\gamma_{L V} \cos \theta_{0}=\gamma_{S V}-\gamma_{S L}$ describes wetting without voltage, namely the voltage $V$ equals to 0 . Then the expression $\frac{\gamma_{S L}-\gamma_{S V}}{\gamma_{L V}}$ can be replaced by $\cos \theta_{0}$ and eqn (16) can be rewritten as

$$
\cos \theta(V)=\cos \theta_{0}+\frac{\varepsilon V^{2}}{2 \gamma_{L V} d} \cdot \frac{1}{1+\frac{d}{R}}
$$

The above derivation is for EWOD on convex spherical surfaces. In the case of a metal substrate with a concave surface (Fig. 2(b)), we can get the expression relating $\theta$ to $V$ and $R$ in the similar way. For EWOD on a concave surface (radius is $R$ ) covered with a dielectric film whose thickness is $d$, the relationship between $\theta$ and $R$ is:

$$
\cos \theta(V)=\cos \theta_{0}+\frac{\varepsilon V^{2}}{2 \gamma_{L V} d} \cdot \frac{1}{1-\frac{d}{R}}
$$

It should be noticed that in the case of EWOD on concave surfaces, the dielectric film is coated on the concave substrate with uniform thickness, so the thickness of the film $d$ must be smaller than the solid radius $R$, namely $\frac{d}{R}<1$.

If taking the curvature (or radius) as an algebraic value, (i.e., concave surfaces have negative curvatures while convex surfaces have positive curvatures), we can get eqn (18) from eqn (17) just substituting $R$ with $-R$. Therefore, for EWOD on spherical surfaces, the relationship among the contact angle, the applied voltage and the surface curvature can be taken as a uniform expression:

$$
\cos \theta(V)=\cos \theta_{0}+\frac{\varepsilon V^{2}}{2 \gamma_{L V} d} \cdot \frac{1}{1 \pm \alpha},
$$

where $\alpha=d / R ;$ i.e., for a convex surface $\cos \theta(V)=\cos \theta_{0}+\frac{\varepsilon V^{2}}{2 \gamma_{L V} d} \cdot \frac{1}{1+\alpha}$, and for a concave surface $\cos \theta(V)=\cos \theta_{0}+\frac{\varepsilon V^{2}}{2 \gamma_{L V} d} \cdot \frac{1}{1-\alpha}$.

Considering that the capacitance of a spherical capacitor per unit area is $c=\frac{\varepsilon}{d(1 \pm \alpha)}$, eqn (19) can be also expressed as

$$
\cos \theta(V)=\cos \theta_{0}+\frac{1}{2} \cdot \frac{c V^{2}}{\gamma_{L V}}
$$

where $c$ is the capacitance per unit area.

\section{EWOD on cylindrical surface}

Consider EWOD on a convex cylindrical surface. The cross section of this case is the same as that of a convex spherical surface, as shown in Fig. 2(a). The average value of the electric field intensity $E(r)$ can be expressed as

$$
\langle E(r)\rangle=\frac{Q}{\varepsilon A_{r}}=\frac{Q}{\varepsilon \beta r} .
$$

Then the voltage can be calculated as the function of $E$

$$
V=\int_{R}^{R+d}\langle E(r)\rangle d r=\langle E(r)\rangle \cdot r \ln \frac{R+d}{d} .
$$

We can get

$$
\frac{d U}{d A}=\int_{R}^{R+d} \frac{1}{2}\left\langle\varepsilon E^{2}\right\rangle \frac{r}{R+d} d r=\frac{\varepsilon V^{2}}{2} \cdot \frac{1}{(R+d) \ln \frac{R+d}{R}}
$$

and

$$
\frac{d W_{B}}{d A}=V \sigma=V \varepsilon E_{R+d}=\frac{\varepsilon V^{2}}{(R+d) \ln \frac{R+d}{R}}
$$

Using eqns (23) and (24), and considering the Young equation, we get the expression of EWOD on a convex cylindrical surface

$$
\cos \theta(V)=\cos \theta_{0}+\frac{\varepsilon V^{2}}{2 \gamma_{L V} d} \cdot \frac{d}{(R+d) \ln \frac{R+d}{R}}
$$

For EWOD on a metal substrate with a concave cylindrical surface, we can get the expression relating $\theta$ to $V$ and $R$ in the similar way. The relationship between $\theta$ and $R$ for EWOD on a concave cylindrical surface is: 


$$
\cos \theta(V)=\cos \theta_{0}+\frac{\varepsilon V^{2}}{2 \gamma_{L V} d} \cdot \frac{d}{(R-d) \ln \frac{R}{R-d}}
$$

If taking the curvature (or radius) as an algebraic value, the relationship among the contact angle, the applied voltage and the surface curvature can be taken as a uniform expression:

$$
\cos \theta(V)=\cos \theta_{0}+\frac{\varepsilon V^{2}}{2 \gamma_{L V} d} \cdot \frac{\alpha}{(1 \pm \alpha) \ln (1 \pm \alpha)}
$$

As the capacitance of a cylindrical capacitor per unit area is $c=\frac{\varepsilon \alpha}{d(1 \pm \alpha) \ln (1 \pm \alpha)}$, eqn (27) can be simplified as

$$
\cos \theta(V)=\cos \theta_{0}+\frac{1}{2} \cdot \frac{c V^{2}}{\gamma_{L V}}
$$

\section{Results and discussions}

In the previous part, we derived EWOD on spherical and cylindrical surfaces. From the derivations above, we find that eqns (20) and (28) are in the same form. Actually, it can be demonstrated that eqn (20) or eqn (28) is always applicable for EWOD on surfaces with various curvatures. The form of eqn (20) or eqn (28) is the same as the EW equation expressed in the form of capacitance, i.e., eqn (5). This is really an interesting finding. For EWOD on curved surfaces under low voltage, once the capacitance per unit area is known, the variation of the contact angle can be predicted. However, it should be noticed that although the extended EW equation can be simplified as the form of capacitance, it is not just simply a problem of capacitor, since the mechanism of EW is still debatable, which has been interpreted in the introduction.

In order to facilitate discussion but without loss of generality, we focus on EWOD on a spherical surface in the following discussion.

Eqn (19) shows that the variation of the contact angle in EWOD is dependent on the surface curvature. From eqn (19), it can be seen that the contact angle is influenced by the initial contact angle $\theta_{0}$, the applied voltage $V$, the liquid-vapor interfacial energy $\gamma_{L V}$, the thickness of the dielectric film $d$, and $\alpha$ which is the ratio of $d$ to $R$. The radius of the droplet seems having no influence on the contact angle for EWOD on curved surfaces. Virtually, from eqn (11), it can be seen that the electric intensity $E$ is affected by $\beta$, which is related to the radius of the droplet; but as $E$ is also affected by the quantity of the electric charge $Q$, which is also related to $\beta$, the influence of $\beta$ cancels out.

EWOD on planar surfaces follows the EW equation, which is different from EWOD on curved surfaces described by eqn (19). However, as EWOD is an objective phenomenon, whose mechanism cannot be changed by the geometry of the metal substrate, eqn (19) and the EW equation are related. Let $R \rightarrow \infty$, or $\alpha \rightarrow 0$, eqn (19) degenerates into the EW equation. In the EW equation, the last term $\eta=\frac{\varepsilon V^{2}}{2 \gamma_{L V} d}$ is a dimensionless number, which is called the EW number. Then the EW equation can be rewritten as

$$
\cos \theta(V)=\cos \theta_{0}+\eta
$$

Considering the influence of the surface curvature, and comparing eqn (19) with the EW equation, we introduce a curvature-modified EW number

$$
\eta_{R}=\frac{\varepsilon V^{2}}{2 \gamma_{L V} d} \cdot \frac{1}{1 \pm \alpha}
$$

Then eqn (19) can be rewritten as

$$
\cos \theta(V)=\cos \theta_{0}+\eta_{R}
$$

The curvature-modified EW number can be expressed as a function of $\eta$ and $\alpha$, and then we can get

$$
\eta_{R}=\eta \cdot \frac{1}{1 \pm \alpha} .
$$

When the thickness of the dielectric film $d$ in the ratio $\alpha=\mathrm{d} / R$ is fixed, $R$ is the only factor that makes $\eta_{\mathrm{R}}$ different from $\eta$.

Let $R \rightarrow \infty$, then $\eta_{R} \rightarrow \eta$, eqn (31) turns into eqn (29) and $\theta$ $\rightarrow \theta_{E W}$. For EWOD on concave surfaces, the radius of the substrate $R$ is larger than the thickness of the dielectric film $d$. For EWOD on convex surfaces, if $R \rightarrow 0$, i.e., $\alpha=\frac{d}{R} \rightarrow \infty$, then $\eta_{R} \rightarrow 0$, which means the induced voltage will have very little influence on the contact angle. Actually in real situations, no matter in experiments or realized applications, the dielectric film is always very thin compared with the substrate, namely $\alpha=\frac{d}{R} \ll 1$.

Comparing EWOD on curved surfaces with that on planar surfaces, we find that at the same initial condition, the contact angle on concave surfaces changes faster while that on convex surfaces changes slower. This is because the EW number is dependent on the surface curvature. In the current case, it means that $\eta_{R}(-R)>\eta>\eta_{R}(R)$. Verheijen et al. ${ }^{35}$ carried out experiments of EWOD on planar surfaces with the parylene- $N$ coating. Referring to their parameters, we suppose $\theta_{0}=120^{\circ}, \gamma_{L V}=72$ $\mathrm{mN} \mathrm{m}{ }^{-1}, \varepsilon_{r}=2.65, d=8 \mu \mathrm{m}$, and get the following curves (the applied voltage ranges from 0 to $300 \mathrm{~V}$ ) in Fig. 4 . As $R$ becomes smaller, or the value of $\alpha$ becomes bigger, the difference becomes much more obvious.

In EWOD experiments on planar surfaces, ${ }^{18}$ the relationship between the contact angle and the applied voltage satisfies the EW equation at low voltage only. At high voltage, the contact angle was found to saturate, say $\theta_{\text {sat }}$ (the saturated contact angle) varies between $30^{\circ}$ and $80^{\circ} .{ }^{17}$ According to Verheijen's experiments, the contact angle saturates around $60^{\circ}$ under $240 \pm 10 \mathrm{~V}$. From Fig. 4, the variation of the contact angle differs with different values of $\alpha$. The difference among the three curves when $\alpha$ equals to 0.2 is distinct, while the curves nearly overlap in the other two situations ( $\alpha=0.02$ and $\alpha=0.002$ ). For $\alpha=0.2$, when $V$ equals to $100 \mathrm{~V}, \theta$ deviates from $\theta_{\mathrm{f}}$ (the contact angle on planar surfaces) at $1.9 \%$ and $-2.8 \%$ on convex and concave surfaces, respectively; when $V$ equals to $150 \mathrm{~V}$, deviations are $4.8 \%$ and $-7.1 \%$; when $V$ is $200 \mathrm{~V}$, deviations are $11.2 \%$ and $-12.0 \%$. When $V$ is $240 \mathrm{~V}$, in which situation the contact angle almost saturates on planar surfaces, the distinction can be more than $10^{\circ}$. Since the thickness of the dielectric film is as small as several microns, the radius of the solid surface is the main factor that influences the contact angle in present experiments. For EWOD 


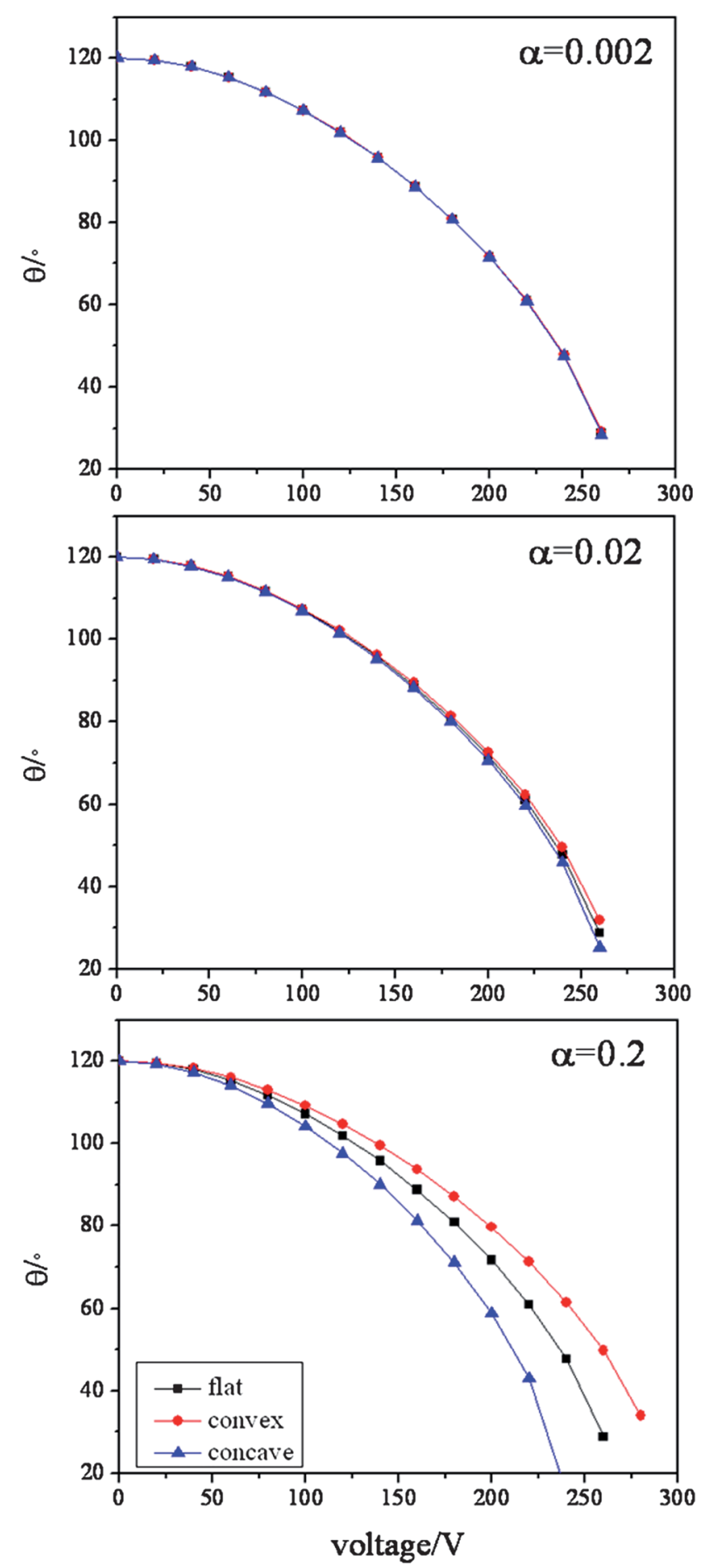

Fig. 4 Contact angle $\theta$ versus the applied voltage $V$ for EWOD on spherical substrate with various $\alpha$, where $\alpha=d / R$. $d$ is fixed, $R$ is the only variable.

on convex solid surfaces, the contact angle changes faster as $R$ becomes larger; for EWOD on concave surfaces, the contact angle changes slower as $R$ becomes larger. The relationship between the contact angle and the radius of the surface is shown in Fig. 5, the parameters of the system are the same as those mentioned above. It should be again emphasized that the radius of the substrate $R$ is larger than the thickness of the dielectric film $d$ for EWOD on concave surfaces, i.e., $R>8 \mu \mathrm{m}$ in this case. In both situations, as $R$ increases, the variation of the contact angle on curved surfaces becomes more close to planar situation. When $R \rightarrow \infty$, eqn (19) turns into the EW equation. The planar surface can be taken as a curved one whose radius is infinite, so the EW equation is a degenerate equation considering the surface curvature effect in a sense. When $R \rightarrow 0$ for convex case, eqn (19) turns into the Young equation and $\theta \rightarrow 120^{\circ}$, in which instance the dielectric film is relatively thick so that nearly all of the electric energy is stored in it.

From eqn (19) and Fig. 5, it can be seen that the surface curvature has influence on the contact angle. At present experimental conditions, $\alpha$ or the ratio of $d$ to $R$ is small which makes the modified term of the EW number closing to 1 . According to our results, the variation of the contact angle on curved surfaces is close to that on planar surfaces, and surface curvature effect is not obvious in this case. This is in accordance with the experimental observation. ${ }^{26,27}$ However, when $R$ becomes very small or EWOD on and below microscale, such as EWOD in CNTs or on graphene substrate, the influence of the surface curvature cannot be neglected. As far as we are concerned, there are few experiments on and below the microscale in the published work, and we would like to deal with it in our future work.

In the end, we try to establish a uniform expression of EWOD on various geometrical surfaces. All the derivations and discussion above do not take the line tension into account. According to relevant studies, ${ }^{31,32}$ the line tension (the orders of magnitude range from $10^{-11}$ to $10^{-6} \mathrm{~N}$ ) works on micro- and nanoscales. If considering the effect of the line tension, by using the method of energy minimization in a similar way as the above derivations (see Appendix for details), then we get

$$
\cos \theta(V)=\cos \theta_{Y}-\frac{\tau \chi}{\gamma_{L V}}+\frac{1}{2} \cdot \frac{c V^{2}}{\gamma_{L V}}
$$

where $\theta_{Y}$ is the Young contact angle, $\tau$ is the line tension, $\chi$ is the geodesic curvature,,$^{33}$ and $c$ is the capacitance per unit area. Eqn (33) was derived under the assumption that the line tension is not affected by the voltage. The effect of voltage on the line tension is the next step for future work. Considering the line tension, wetting on spherical surface is shown in Fig. 6. At the three-phase contact line, the surface tensions and the line tension work together to maintain the equilibrium of the tangent force (the direction of the solid-liquid or solid-vapor surface tension). When a voltage is applied, an electrostatic force will contribute to the balance of the tangent force. However, the value and the direction of the electrostatic force are still unclear, further investigation is necessary.

\section{Conclusions}

An extended EW equation for EWOD on curved surfaces is proposed using the approach of energy minimization. In the equation, a curvature-modified EW number is introduced to describe the surface curvature effect. It is found that the surface curvature has significant influence on the contact angle, especially when the system is on and below microscale. Since a curved surface can be either convex or concave, the variation of the contact angle is different. While the variation of the contact angle 

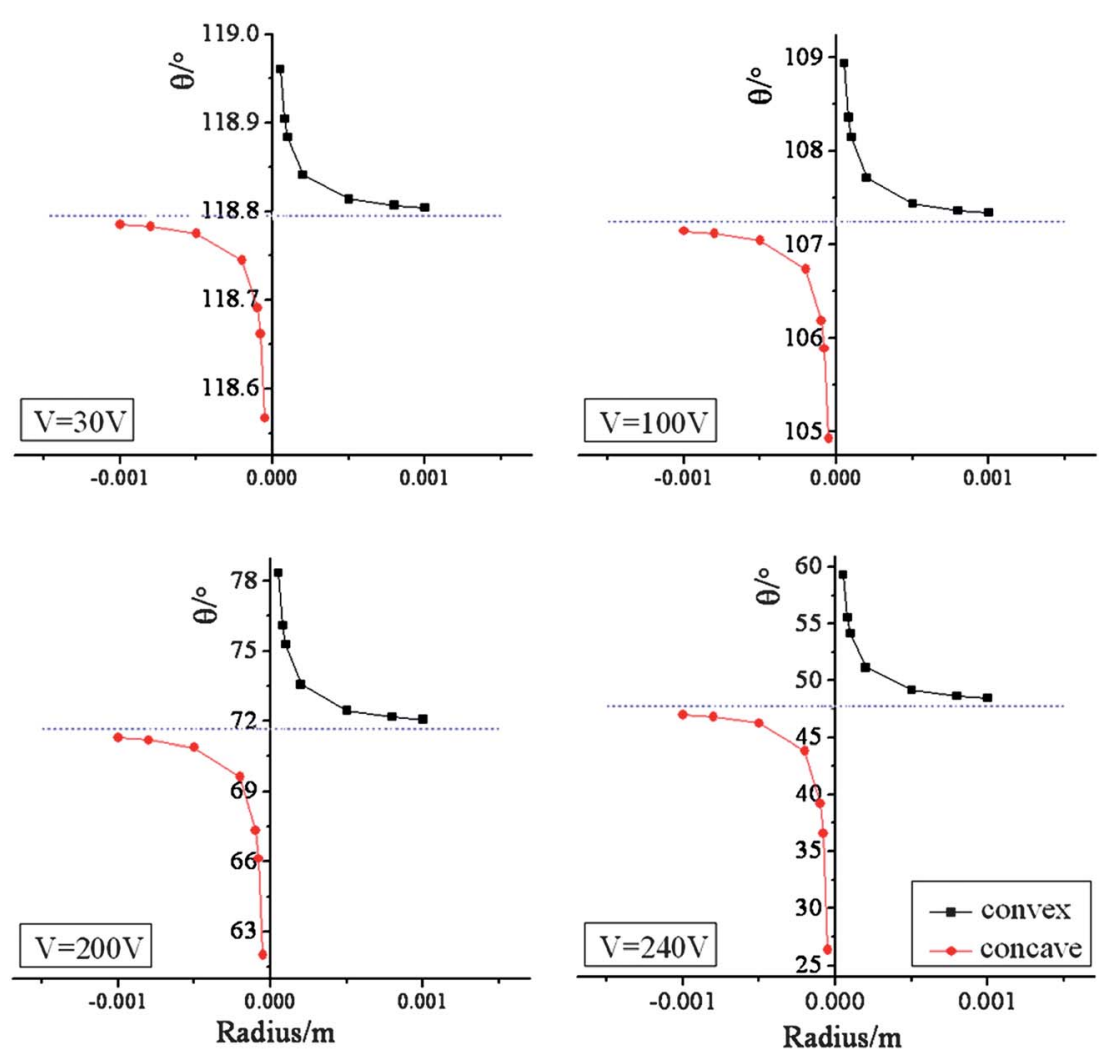

Fig. 5 Contact angle $\theta$ versus the radius of the spherical substrate under different applied voltage $V$. For convex surfaces, $\theta \rightarrow 120^{\circ}$ when $R \rightarrow 0$ and $\theta$ $\rightarrow \theta_{E W}$ when $R \rightarrow \infty$; for concave surfaces, $d / R<1$ so that $R>8 \mu \mathrm{m}$.

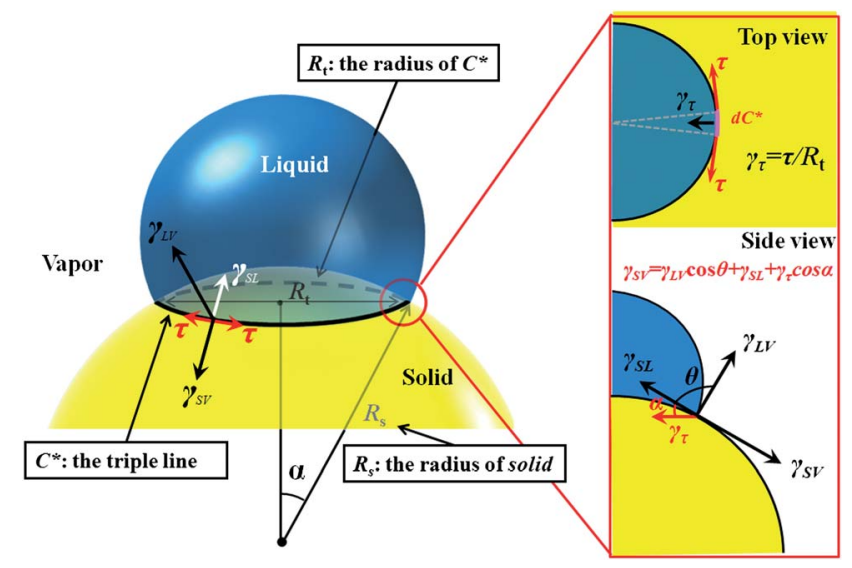

Fig. 6 Schematic of wetting on spherical surface considering the line tension. The upper right represents the resultant force $\left(\gamma_{\tau}\right)$ of the line tension at the contact point from the top view; the lower right shows the forces from the side view. The balance of the tangent forces results in the equation $\gamma_{S V}=\gamma_{L V} \cos \theta+\gamma_{S L}+\gamma_{\tau} \cos \alpha$.

on convex surfaces decreases compared with that on planar surfaces, it increases on concave surfaces. This means that concave surfaces can enhance wettability better than convex surfaces when applying the same voltage. Afterwards, considering that the line tension will work at micro- and nanoscales, an extended expression for EWOD on curved surfaces is proposed. This significant surface curvature effect on improving the wettability of the system can provide a theoretical key for future applications of flexible electronics.

\section{Appendix}

\section{Derivation of extended electrowetting equation considering the line tension}

Take the spherical case for example, the equation that considering the line tension can be derived as follows.

With an infinitesimal increase of the base area $\mathrm{d} A$, the free energy $\mathrm{d} F$ of the system changes:

$$
d F=\gamma_{S L} d A-\gamma_{S V} d A+\gamma_{L V} d A \cos \theta+\tau d l+d U-d W_{B},
$$

where $\mathrm{d} l$ is the infinitesimal length increase of the three-phase contact line. $\tau \mathrm{d} l$ equals to $\frac{\tau}{R_{t}} \cos \alpha \cdot d A=\frac{\tau}{R_{t}} \sqrt{1-\left(\frac{R_{t}}{R_{s}}\right)^{2}} \cdot d A$, where $R_{\mathrm{t}}$ is the radius of the triple line and $R_{\mathrm{s}}$ is the radius of the solid surface, as illustrated in Fig. 6. In order to be stable, the free energy should reach a minimum, then we get the equation:

$$
\gamma_{S L}-\gamma_{S V}+\gamma_{L V} \cos \theta+\frac{\tau}{R_{t}} \sqrt{1-\left(\frac{R_{t}}{R_{s}}\right)^{2}}+\frac{d U}{d A}-\frac{d W_{B}}{d A}=0 .
$$

The terms of $\mathrm{d} U / \mathrm{d} A$ and $\mathrm{d} W_{\mathrm{B}} / \mathrm{d} A$ are the same as those in eqns (14) and (15), i.e., $\frac{d U}{d A}=\frac{\varepsilon V^{2}}{2 d} \cdot \frac{R}{R+d}$ and $\frac{d W_{B}}{d A}=V \sigma=$ $V \varepsilon E_{R+d}=\frac{\varepsilon V^{2} R}{(R+d) d}$. Substituting $\mathrm{d} U / \mathrm{d} A$ and $\mathrm{d} W_{\mathrm{B}} / \mathrm{d} A$ into eqn (A2), and using the Young equation, then we can get 


$$
\cos \theta(V)=\cos \theta_{0}+\frac{\tau}{\gamma_{L V}} \frac{\sqrt{1-\left(R_{t} / R_{s}\right)^{2}}}{R_{t}}+\frac{\varepsilon V^{2}}{2 \gamma_{L V} d} \cdot \frac{1}{1+\frac{d}{R}} .
$$

According to ref. 33, $\chi=\frac{\sqrt{1-\left(R_{t} / R_{s}\right)^{2}}}{R_{t}}$ is the geodesic curvature of the triple line in a spherical case, so eqn (A3) can be simplified as

$$
\cos \theta(V)=\cos \theta_{Y}-\frac{\tau \chi}{\gamma_{L V}}+\frac{1}{2} \cdot \frac{c V^{2}}{\gamma_{L V}}
$$

\section{Acknowledgements}

This work was jointly supported by the National Natural Science Foundation of China (NSFC, Grant Nos. 11072244, 60936001, 11021262 and 11011120245), and the Instrument Developing Project of the Chinese Academy of Sciences (Grant No. Y2010031).

\section{References}

1 M. G. Pollack, A. D. Shenderov and R. B. Fair, Lab Chip, 2002, 2, 96-101.

2 S. K. Cho, H. Moon and C. J. Kim, J. Microelectromech. Syst., 2003, 12, 70-80.

3 I. Barbulovic-Nad and A. R. Wheeler, Encyclopedia of Micro- and Nanofluidics, Springer, Heidelberg 2008.

4 S. Kuiper and B. H. W. Hendriks, Appl. Phys. Lett., 2004, 85, 11281130.

5 B. Berge and J. Peseux, Eur. Phys. J. E: Soft Matter Biol. Phys., 2000, 3, 159-163.

6 J. A. Rogers, Z. Bao, K. Baldwin, A. Dodabalapur, B. Crone, V. R. Raju, V. Kuck, H. Katz, K. Amundson, J. Ewing and P. Drzaic, Proc. Natl. Acad. Sci. U. S. A., 2001, 98, 48354840.
7 H. You and A. J. Steckl, J. Adhesion Sci. Technol., 2011, DOI: 10/ 1163/156856111X600244.

8 J. T. Feng, F. C. Wang and Y. P. Zhao, Biomicrofluidics, 2009, 3, 022406.

9 R. A. Hayes and B. J. Feenstra, Nature, 2003, 425, 383-385.

10 D. Y. Kim and A. J. Steckl, ACS Appl. Mater. Interfaces, 2010, 2, 3318-3323.

11 Q. Z. Yuan and Y. P. Zhao, J. Am. Chem. Soc., 2009, 131, 63746376.

12 X. C. Qin, Q. Z. Yuan, Y. P. Zhao, S. B. Xie and Z. F. Liu, Nano Lett., 2011, 11, 2173-2177.

13 Q. Z. Yuan and Y. P. Zhao, Phys. Rev. Lett., 2010, 104, 246101.

14 G. Lippmann, Ann. Chim. Phys., 1875, 5, 494-549.

15 L. Minnema, H. A. Barneveld and P. D. Rinkel, IEEE Trans. Electr. Insul., 1980, 15, 461-472.

16 G. Beni and S. Hackwood, Appl. Phys. Lett., 1981, 38, 207-209.

17 F. Mugele and J. C. Baret, J. Phys.: Condens. Matter, 2005, 17, 705774.

18 B. Berge, Comptes rendus de l'Académie des sciences. Série II, 1993, 317, 157-163.

19 C. Quilliet and B. Berge, Curr. Opin. Colloid Interface Sci., 2001, 6, 34-39.

20 J. Berthier, Microdrops and Digital Microfluidics, William Andrew Publishing, New York, 2008.

21 R. Gupta, G. K. Olivier and J. Frechette, Langmuir, 2010, 26, $11946-$ 11950.

22 R. Digilov, Langmuir, 2000, 16, 6719-6723.

23 T. B. Jones, Langmuir, 2002, 18, 4437-4443.

24 K. H. Kang, Langmuir, 2002, 18, 10318-10322.

25 F. Mugele, Soft Matter, 2009, 5, 3377-3384.

26 S. K. Fan, H. Yang and W. Hsu, Lab Chip, 2011, 11, 343-347.

27 M. Abdelgawad, S. L. S. Freire, H. Yang and A. R. Wheeler, Lab Chip, 2008, 8, 672-677.

28 J. Y. Chen, A. Kutana, C. P. Collier and K. P. Giapis, Science, 2005, 310, 1480-1483.

29 T. Young, Philos. Trans. R. Soc. London, 1805, 95, 65-87.

30 C. W. Extrand and S. I. Moon, Langmuir, 2008, 24, 9470-9473.

31 J. K. Berg, C. M. Weber and H. Riegler, Phys. Rev. Lett., 2010, 105, 076103.

32 A. Marmur and B. Krasovitski, Langmuir, 2002, 18, 8919-8923.

33 G. Wolansky and A. Marmur, Langmuir, 1998, 14, 5292-5297.

34 E. Bormashenko, J. Phys. Chem. C, 2009, 113, 17275-17277.

35 H. J. J. Verheijen and M. W. J. Prins, Langmuir, 1999, 15, 66166620 . 